\title{
The Metal Hydride Problem of Computational Chemistry: Origins and Consequences
}

\author{
Moltved, Klaus August; Kepp, Kasper Planeta
}

Published in:

Journal of Physical Chemistry Part A: Molecules, Spectroscopy, Kinetics, Environment and General Theory

Link to article, DOI:

10.1021/acs.jpca.9b02367

Publication date:

2019

Document Version

Peer reviewed version

Link back to DTU Orbit

Citation (APA):

Moltved, K. A., \& Kepp, K. P. (2019). The Metal Hydride Problem of Computational Chemistry: Origins and Consequences. Journal of Physical Chemistry Part A: Molecules, Spectroscopy, Kinetics, Environment and General Theory, 123(13), 2888-2900. https://doi.org/10.1021/acs.jpca.9b02367

\section{General rights}

Copyright and moral rights for the publications made accessible in the public portal are retained by the authors and/or other copyright owners and it is a condition of accessing publications that users recognise and abide by the legal requirements associated with these rights.

- Users may download and print one copy of any publication from the public portal for the purpose of private study or research.

- You may not further distribute the material or use it for any profit-making activity or commercial gain

- You may freely distribute the URL identifying the publication in the public portal 


\section{The Metal Hydride Problem of Computational Chemistry: Origins and Consequences}

Klaus A. Moltved and Kasper P. Kepp*

Technical University of Denmark, DTU Chemistry, Building 206, 2800 Kgs. Lyngby, DK Denmark. *Phone: +045 452524 09. E-mail: kpj@kemi.dtu.dk

\section{Abstract}

Formation and breaking of metal-hydrogen bonds are central to many important catalytic processes such as transition-metal catalyzed ammonia synthesis, hydrogenation reactions, and water splitting, and thus require an adequate theoretical description. We studied a dataset of all $30 \mathrm{M}-\mathrm{H}$ and $30 \mathrm{M}^{+}-\mathrm{H}$ bonds of the 3d, 4d, and 5d transition series; 50 of these systems have experimentally known bond dissociation enthalpies (BDE); to probe both the limit of low and high coordination number, we also studied a dataset of $19 \mathrm{ML}_{n} \mathrm{H}$ complexes. The BDEs were computed using Hartree-Fock (HF), MP2, CCSD, CCSD(T), and ten diverse density functionals including local, GGA, hybrid GGA, meta hybrid, range-separated and double hybrids. Our ten most important findings are: 1) HF fails completely to describe the metal hydrogen bond due to its lack of static correlation; 2) this makes post-HF methods such as MP2 and even CCSD(T) perform worse than many density functionals; 3) DFT requires much more HF exchange ( $\sim 35 \%$ on average) to describe the pure $\mathrm{M}-\mathrm{H}$ bonds than to describe other metal ligand bonds $(0-20 \%)$; 4) we design a test to determine if self-interaction error (SIE) is important by correlating DFT errors against a one-electron SIE metric; 5) we show that SIE correlates directly with the DFT errors and thus causes most of the problem; 6) HF-DFT cannot handle these systems because the HF method is too pathological already at the density level; 7) instead, we define and apply a simple metric of electronic abnormality as the difference in 
PBE energy computed at the self-consistent PBE0 and SVWN densities; this metric gives appropriate spread and effectively captures density-derived errors; 8) the low electronegativity of the metal enforces a diffuse hydride-like electron density, which make the metal hydrides primary examples of many-electron systems exhibiting SIE already at equilibrium geometries; 9) in the coordinatively saturated $\mathrm{ML}_{n} \mathrm{H}$ systems, much less HF exchange is required, i.e. the HF exchange requirements vary drastically with coordination number. Accordingly, DFT is unbalanced for any catalytic process involving both $\mathrm{M}-\mathrm{H}$ and $\mathrm{M}-\mathrm{L}$ bonds and changing coordination numbers; 10) importantly, the range-separated and double-hybrid functionals CAM-B3LYP and B2PLYP alone perform well for both M-H and M-L systems and in both limits of low and high coordination number, and at least as well as $\operatorname{CCSD(T).~This~lends~hope~}$ to a balanced treatment of computational chemistry for all types of M-L bonds at variable coordination number, as required for real catalytic reactions.

Keywords: Metal hydrogen bond, DFT, coupled-cluster, electron correlation, self-interaction error, transition metal, catalysis, density error. 


\section{Introduction}

Many important processes, such as transition-metal-catalyzed water splitting, the Haber-Bosch process, and hydrogenation reactions, involve the transient formation and breaking of metalhydrogen bonds $(\mathrm{M}-\mathrm{H})^{1,2}$. These processes are studied computationally and depend directly on the energy of the metal-hydrogen bond, which accordingly feature prominently e.g. in semiquantitative models of catalysis. ${ }^{3,4}$ It is of major importance to ensure that theoretical chemistry accurately describes these chemical bonds, and we need to understand the sources and magnitudes of the errors involved.

Density functional theory (DFT) is the method of choice for most problems in chemistry and catalysis due to the necessary tradeoff between accuracy and computational cost ${ }^{5-7}$. It was found long ago ${ }^{8}$ and recently confirmed for many more functionals ${ }^{9,10}$ that DFT commonly fails dramatically at describing the chemical bonds between transition metals and a hydrogen atom. Questions that immediately arise are: 1) How are these bonds special compared to other chemical bonds? 2) Why is this important? 3) How large are the computational errors? 4) What are the potential consequences for theoretical catalysis? 5) Which remedies exist?

Despite the massive use of DFT for understanding and improving catalytic reactions ${ }^{11,12}$, it is very hard to assess computational accuracy, because systematic benchmark data are scarce. For example, a recently compiled experimental benchmark data set of adsorption energies $^{13}$, although clearly a step in the right direction, contains 34 data points in total and only a handful of data points for each type of adsorbed molecule, while statistical significance probably requires 10-20 data points per adsorbing molecule on different catalysts. To understand and improve computational catalysis, we have argued for a bottom-up strategy starting from small systems that reflect the essential pure M-L bond without diverse modulating effects of other ligands. Experimental data for metal-ligand (M-L) bond dissociation enthalpies 
(BDE) in diatomic molecules are more generic and much more abundant and systematic, thus enabling statistically meaningful conclusions that cannot be obtained from studies of large systems. ${ }^{10,14-16}$. Furthermore, the experimental BDEs are much less noisy than other data as they reflect gas-phase enthalpies without complications of dispersion effects, solvent effects, and entropy effects, and worse, the combinations of these, e.g. solvent dispersion and polarization, which contribute substantial noise to other benchmark data and prevent accurate conclusions to be drawn.

A central point in these efforts is to identify the accuracy bottlenecks for full catalytic process. Metal-catalyzed reactions usually involve changes in the coordination number and type of ligands. The M-L diatomic molecules represent the pure bonds and the limit of low coordination number, and the impact of ligand modulations needs to be accounted for separately. We need to understand how transferable DFT is when applied to the diverse situations of low and high coordination numbers and bonds modulated by diverse ligands. Recent full-cycle benchmarking on a simple model of the Haber-Bosch process showed that different functionals fail for different steps of the catalytic cycle that are electronically distinct. ${ }^{16}$ The M-H bonds were particular problematic. The specialness of M-H bonds has been explored in other contexts ${ }^{1}$. Most M-L bonds are typically optimally described by $10-20 \%$ HFexchange, but this fraction importantly varies with ligand type. ${ }^{10,15,17}$ Accordingly, we suspect that DFT is fundamentally unbalanced when applied to both $\mathrm{M}-\mathrm{H}$ and other M-L bonds, as in e.g. $\mathrm{H}_{2}$ activation, ammonia synthesis, hydrogenation reactions, or hydrogen evolution by transition metal catalysts, with either a large error in the M-H or M-L states. By unbalanced we mean that DFT has non-uniform accuracy for different categories of electronic systems and processes. We suspect that this issue also arises with respect to changes in coordination number, which varies from low to high in real processes. 
To understand the $\mathrm{M}-\mathrm{H}$ bond in systematic detail without bias to any metal or $\mathrm{d}^{\mathrm{q}}$ configuration, we studied all the 30 neutral $\mathrm{M}-\mathrm{H}$ and 30 cationic $\mathrm{M}^{+}-\mathrm{H}$ systems involving all combinations of the 3d, $4 \mathrm{~d}$ and $5 \mathrm{~d}$ transition metals. Experimental BDEs are available for 50 of these 60 systems, enabling a statistically valid assessment of the performance of DFT. To assess also the limit of high coordination number, we computed the BDEs of 19 coordinatively saturated $\mathrm{ML}_{n} \mathrm{H}$ complexes with $\mathrm{M}=\mathrm{Co}, \mathrm{Cr}, \mathrm{Fe}, \mathrm{Hf}, \mathrm{Ir}, \mathrm{Mn}, \mathrm{Mo}, \mathrm{Ni}, \mathrm{Os}, \mathrm{Pt}, \mathrm{Re}, \mathrm{Rh}, \mathrm{V}, \mathrm{W}$, or Zr. A representative group of density functionals was studied in comparison to HF, MP2, CCSD, and CCSD(T) wave function methods, and we analyzed the DFT errors, the HF-related errors and the density errors. 


\section{Methods}

Computational details. Computations were performed using the Turbomole software, version 7.0. ${ }^{18,19}$, except the CAM-B3LYP energies, which were computed using Gaussian $16 .^{20}$ Computations were converged to $10^{-6}$ au, using the resolution of identity approximation ${ }^{21}$. BDEs were calculated for all the 30 neutral $\mathrm{M}-\mathrm{H}$ and 30 cationic $\mathrm{M}^{+}-\mathrm{H}$ systems of the $3 \mathrm{~d}, 4 \mathrm{~d}$ and $5 \mathrm{~d}$ transition metals. Optimized geometries for the 60 diatomic systems were obtained using the BP86 ${ }^{22,23}$ functional and the def2-TZVPP ${ }^{24}$ basis set, with optimized bond lengths given in Table S1. This protocol gives average errors in M-L bond lengths of $0.02 \AA^{25}$ which is close to optimal at the current state of the art. Single-point energies for calculating the BDEs were computed with the def2-QZVPPD ${ }^{24}$ basis set for the metals (Sc-Zn, Y-Cd and La-Hg) and aug-cc-pV5Z ${ }^{8}$ for hydrogen to ensure that the diffuse electron-rich hydride state was adequately described.

Spin states and spin-annealing to avoid meta-stable configurations. In all cases, regardless of the known ground state, we ensured adequate convergence by using a protocol of “spin annealing”, i.e. starting the geometry optimization from a higher spin multiplicity than expected for the system (for most systems $M_{\mathrm{S}}+2$ ) and, after energy calculation, decreasing the spin multiplicity in steps of 2 by converting one alpha electron to beat electron in the control input file of Turbomole. The alternative optimization starting directly from the expected spin state commonly leads to meta-stable configurations that are not completely spin polarized, because the start orbitals are very approximate. This is true for DFT but very much also for post-HF methods, since a CCSD(T) calculation on a meta-stable HF is completely erroneous. The spin multiplicities of the ground states used were either experimentally known or, if not available, those determined to have the lowest energy upon geometry optimization using the 
procedure described above. The spin states used can be found in Table S2 for easy reproducibility. Table S3 shows the experimental spin state. As can be seen, the computational protocol correctly identifies the ground state spin for all cases where experiment is available, giving us trust in the assignment of the remaining spin states as well. Table $\mathbf{S} 4$ shows the experimental spin states of atoms and ions available in NIST ${ }^{27}$. In five systems there was another spin state within $20 \mathrm{~kJ} / \mathrm{mol}$ of the identified ground state; these five cases are listed in Table S5. For these systems, we are uncertain about the assignment of the spin state, but the energy effect on the BDE amounts to maximally $16 \mathrm{~kJ} / \mathrm{mol}$ and as they constitute only $10 \%$ of all systems where experimental BDEs can be used for benchmarking, the impact on the final reported mean absolute errors (MAE) will be maximally $1.6 \mathrm{~kJ} / \mathrm{mol}$, if all differences work to change the MAE in the same direction. Thus, the assignment of applied spin states is robust to the conclusions and results stated later in this work.

Zero-point vibrational and relativistic corrections to the electronic energies. Zero point energies (ZPE) were calculated from a frequency analysis of the optimized ground state and can be seen in Table S6. For the 3d metal hydrides, scalar-relativistic effects were estimated using the sum of the Darwin term and the expectation value of $P^{4}$ from B3LYP; the corresponding correction to the BDE can be seen in Table S7. For the $4 \mathrm{~d}$ and $5 \mathrm{~d}$ transition metal hydrides the scalar-relativistic effects were approximated by using effective core potentials. The effect of spin-orbit coupling (SOC) was estimated by performing a DouglasKroll-Hess B3LYP energy calculation of 4th order for the 3d metal hydrides with the dhfQZVPP-2c basis set ${ }^{28}$. The effect of SOC for the $4 \mathrm{~d}$ and $5 \mathrm{~d}$ metal hydrides was estimated by a two-component calculation including ECP calling the Turbomole keyword soghf also using the dhf-QZVPP-2c basis set. The SOC corrections to the BDE are compiled in Table S8. Please note that the two different treatments of the SOC give different SOC corrections. The DKHbased SOC corrections for the 3d systems is probably overestimated by a factor of 2 when 
using the all electron treatment, whereas the SOC for the $4 \mathrm{~d}$ and $5 \mathrm{~d}$ system may be slightly too small when using ECPs ${ }^{28,29}$. However this does not influence our conclusions since the largest SOC correction for the $3 \mathrm{~d}$ systems is $2.5 \mathrm{~kJ} / \mathrm{mol}$, i.e. even a $100 \%$ overestimation would cause a maximum error of $2.5 \mathrm{~kJ} / \mathrm{mol}$ in the worst case. For the $4 \mathrm{~d}$ and $5 \mathrm{~d}$ systems the largest SCO correction is $-23.7 \mathrm{~kJ} / \mathrm{mol}$; a $20 \%$ underestimation would introduce an error of $\sim 5 \mathrm{~kJ} / \mathrm{mol}$. These errors are upper estimates for the largest SOC values, and thus the effect on the average data used in this paper's analysis will be substantially smaller.

Methods studied. The functionals used for the energy calculations are compiled in Table 1. We were not interested in performing a benchmark of DFT broadly as this has already been done previously for a smaller set of $\mathrm{M}-\mathrm{H}$ systems $\mathrm{s}^{9,10,15,25,30}$. We wanted to explore how transferable ("universal”) DFT is, i.e. the generality, magnitude, and origin of the failure for metal hydrides across all three d-blocks and all d-electron configurations, and thus studied the most complete experimental data set of M-H BDEs so far. B2PLYP ${ }^{31}$, BHLYP ${ }^{32}$, CAM-

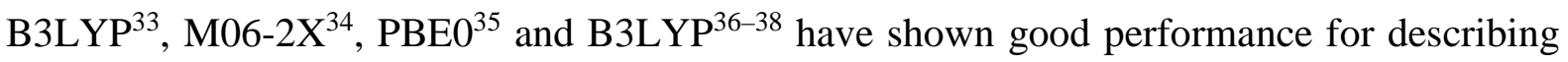
3d metal hydrides ${ }^{10}$. $\mathrm{PBE}^{35}$ and $\mathrm{RPBE}^{39}$ were studied because of their widespread use in theoretical catalysis. BLYP 37,40 was used to provide non-hybrid comparison to the $20 \%$ HFexchange in the much-used hybrid functional B3LYP. SVWN was included to see the behavior of a strictly local non-GGA functional. In addition to these density functionals in Table 1, BDEs were also computed for all 60 molecular systems using HF, MP2, CCSD and CCSD(T) and the same basis sets as for DFT for direct comparison. The raw single point electronic energies are compiled for reproducibility purposes in Tables S9-S11 for the atoms and Tables S12-S14 for the diatomic metal hydrides. 
Table 1. The ten exchange-correlation functionals studied, their functional type, the amount of Hartree-Fock exchange (if any), and key references.

\begin{tabular}{|l|l|l|l|}
\hline Functional & Type & \% HF exchange & References \\
\hline SVWN & LSDA & 0 & 41,42 \\
\hline BLYP & GGA & 0 & 40,43 \\
\hline PBE & GGA & 0 & 35 \\
\hline RPBE & GGA & 0 & 39 \\
\hline B3LYP & Hybrid GGA & 20 & $36-38$ \\
\hline PBE0 & Hybrid GGA & 25 & 35,44 \\
\hline BHLYP & Hybrid GGA & 50 & 32 \\
\hline CAM-B3LYP & Range-separated hybrid & $19-65$ & 33 \\
\hline M06-2X & Hybrid meta GGA & 54 & 34 \\
\hline B2PLYP & Double Hybrid & 53 & 31 \\
\hline
\end{tabular}

Calculation of BDEs and errors. The BDEs were calculated as:

$$
B D E(M-H)=E(M)+E(H)-E(M-H)-E_{Z P E}+E_{\text {scalar }}+E_{S O C}+3.7 \mathrm{~kJ} / \mathrm{mol}
$$

Here, $E(M), E(H)$ and $E(M-H)$ are the single point energies of the metal, $\mathrm{H}$ and $\mathrm{M}-\mathrm{H}$ respectively. $E_{Z P E}$ is the computed zero-point energy of the metal hydride. $E_{\text {scalar }}$ is the scalar relativistic energy correction previously mentioned. $E_{S O C}$ is the computed SOC correction for the metal hydride and $3.7 \mathrm{~kJ} / \mathrm{mol}$ corresponds to 3/2 RT at $298 \mathrm{~K}$ which is used to convert the BDE from $0 \mathrm{~K}$ to $298 \mathrm{~K}$. The 60 BDEs calculated for the ten functionals of Table 1, and for HF, MP2, CCSD and CCSD(T) are compiled in Tables S15-S17.

The signed errors (SE) discussed in this work were calculated by Equation 2:

$$
S E=B D E(M H)_{\text {computed }}-B D E(M H)_{\text {experimental }}
$$


where $B D E(M H)_{\text {computed }}$ is the BDE calculated from Equation 1, and $B D E(M H)_{\text {experimental }}$ is the experimental BDE from Table $\mathbf{S 1 8}$ (black numbers and $\mathrm{MnH}=126 \mathrm{~kJ} / \mathrm{mol}$ ).

Experimental data and sensitivity analysis. For 20 of the neutral M-H systems and 30 of the cationic $\mathrm{M}^{+}$-H systems, experimental BDEs are available from the CRC Handbook of Chemistry and Physics ${ }^{45}$ and from additional literature as seen in Table S18, with reported experimental errors in Table S19. In most cases the experimental BDEs are similar within their reported uncertainty. However, there are a few cases with significant differences in reported values, most notably $\mathrm{MnH}$. As previously discussed ${ }^{10}$ the value reported in CRC Handbook ${ }^{45}$ for $\mathrm{MnH}$ is unreasonable, and the alternative value of $126 \mathrm{~kJ} / \mathrm{mol}$ of Table $\mathbf{S 1 8}$ was thus used. To test whether the choice of experimental data used affect our results, we performed a sensitivity test by comparing the difference between experimental and computed BDEs using as experimental test set either 1) the CRC handbook data, 2) the average values of all the experimental data in Table S18 (counting duplicates only once), or 3) the values with the largest reported deviations from the CRC handbook data. The results of this analysis in the form of the mean signed error (MSE), MAE, the standard deviation of the signed error and absolute error, and the maximum and minimum errors, are shown for all alternative comparisons in Tables S20-S23. The use of average experimental values had a very small impact on the errors (maximally $1.5 \mathrm{~kJ} / \mathrm{mol}$ for the MAEs), but this changes ranking for some of the similarly performing methods. ${ }^{10}$ Using the data with the largest deviation worsened performance for all methods, including $\operatorname{CCSD}(\mathrm{T})$, consistent with the use of less correct benchmark numbers. The smallest errors were seen using the CRC Handbook data but changing $\mathrm{MnH}$ to the consensual $126 \mathrm{~kJ} / \mathrm{mol}$ reported by two other sources (Table S23). The data set used in the analysis is thus that of Table S23. 
BDEs of M-H bonds of coordination complexes with more ligands. To investigate also the limit of high coordination number, 19 complexes of the type $\mathrm{ML}_{n} \mathrm{H}$ ( $\mathrm{L}$ can be several types of ligands) were treated with the same functionals as for the diatomic systems (Table 1). The experimental M-H BDEs of these complexes were taken from the CRC Handbook ${ }^{45}$ (Table S24). The basis set used for these larger systems was def2-QZVPP ${ }^{24}$ for both metals and ligand atoms. Since these systems have intramolecular dispersion interactions, the DFT-D3 correction $^{46}$ was included in the calculation (for RPBE and M06-2X the dispersion correction for B3LYP was used). Thus the BDE of the $\mathrm{ML}_{n} \mathrm{H}$ systems was calculated using Equation 3:

$$
B D E\left(M L_{n}-H\right)=E\left(M L_{n}\right)+E(H)-E\left(M L_{n} H\right)-E_{Z P E}+3.7 \mathrm{~kJ} / \mathrm{mol}
$$

$E\left(M L_{n}\right)$ is the energy of $M L_{n} H$ with a hydrogen removed. $E\left(M L_{x} H\right)$ is the energy of $M L_{n} H$, with computed numbers in Tables S25-S26. The single point energies can be found in Tables S27-S28. The BDEs calculated using equation 3 can be found in Table S29-S30. The D3 dispersion corrections and $E_{Z P E}$ values from frequency calculation are compiled in Table S31. These 19 complexes constitute a relatively homogenous data set with similar ligands, generally cyclopentadienyls, carbonyls, and phosphines, and sample many metals across the d-block (M = Co, Cr, Fe, Hf, Ir, Mn, Mo, Ni, Os, Pt, Re, Rh, V, W, or Zr).

HF-DFT energies, abnormality metrics, and SIE metrics. Burke's group has advocated $^{47,48}$ using a DFT non-consistent single-point energy calculation on the selfconsistent HF density (HF-DFT) for abnormal electronic systems. Since the transition metal hydrides are expected (and showed below) to be challenging, a test of this remedying protocol was applied. PBE, PBE0 and RPBE calculations were carried out on the HF densities (referred to as HF-PBE, HF-PBE0 and HF-RPBE subsequently) by setting the number of iterations for the SCF procedure to 1 and changing the density convergence criteria to $10^{7}$ au. The singlepoint energies obtained in this way can be seen in Tables S10 and S13. Following the 
sensitivity protocol that we proposed ${ }^{49}$, Sim et al. ${ }^{50}$ suggested a simpler metric $S\left(\tilde{E}_{X C}\right)$ for quantifying abnormality that involves only the densities from a HF and a local SVWN ${ }^{41,42}$ calculation with the non-consistent energies computed by PBE (referred to as SVWN-PBE below). The resulting values of the $S\left(\widetilde{E}_{X C}\right)$ metric for the calculated BDEs can be seen in Table S32, together with our pure-DFT metric, which involves PBE0 instead of HF.

The SIE is well-defined for one electron by Zhang and Yang ${ }^{51}$. Quantifying the $N$ electron SIE is however less obvious ${ }^{51}$. Some approaches have been developed e.g. by Lundberg et al. ${ }^{52}$ and Ruzsinszky et al. ${ }^{53}$ Several functionals seek to eliminate the 1-electron SIE, but typically with a cost of accuracy, although e.g. the newer MYC3 and rCAM-B3LYP show promise for the description of SIE-prone systems. ${ }^{54}$ In the present paper we used the archetypical SIE-prone $\mathrm{H}_{2}{ }^{+}$dissociation to establish the 1-electron SIE based on the equation of Zhang and Yang ${ }^{51}$, calculated at $10 \AA$ separation of the hydrogen atoms. We find this metric to be very robust and it spreads the functionals effectively as required. We used this value as our measure of the inherent SIE of the studied functionals (Table S33). 


\section{Results and Discussion}

Errors of modeled M-H BDEs. Considering the importance of the M-H bond to many catalytic processes and the limited benchmarks available in particular for catalytically important $4 \mathrm{~d}$ and $5 \mathrm{~d}$ metals, we wanted to understand the ability of theoretical methods to describe this bond using the available experimental data. Suspecting the major importance of the HF description for these systems ${ }^{10}$, we selected a group of density functionals with specific emphasis on various design types ranging from local DFT (SVWN), via gradient-corrected non-hybrids (PBE, BLYP, RPBE), to hybrids and meta hybrids with variable amounts of HF exchange (B3LYP, PBE0, M06-2X), double hybrids (B2PLYP), and range-separated hybrids (CAM-B3LYP). We also computed the BDEs using HF, MP2, CCSD, and CCSD(T) at the same def2-QZVPP/aug-cc-pV5Z basis set level for direct comparison.

Figure 1 shows the performance for the ten selected functionals, together with HF, MP2, CCSD and CCSD(T). Figure 1A shows the MAE for the methods, whereas Figure 1B shows the MSE. The same results divided into $\mathrm{M}-\mathrm{H}$ and $\mathrm{M}^{+}-\mathrm{H}$ systems separately are shown in Figure S1 and Figure S2; we find that they show similar behavior and are thus treated in combination in the following analysis. The HF description is of interest because the systems require hybrids with a large HF fraction for good performance. The HF method (black bars) underbinds on average by $99 \mathrm{~kJ} / \mathrm{mol}$ and has a similar MAE, because this underbinding is very consistent across all three d-transition series. This finding resembles that seen for other transition metal bonds and tells us that HF is very inadequate for these systems because of the correlation resulting from close-lying d-configurations, which are not described by the HF determinant wave function. Thus, even though the pure $\mathrm{M}-\mathrm{H}$ bonds require much more $\mathrm{HF}$ exchange in the DFT treatment, the HF theory itself is inherently pathological. 


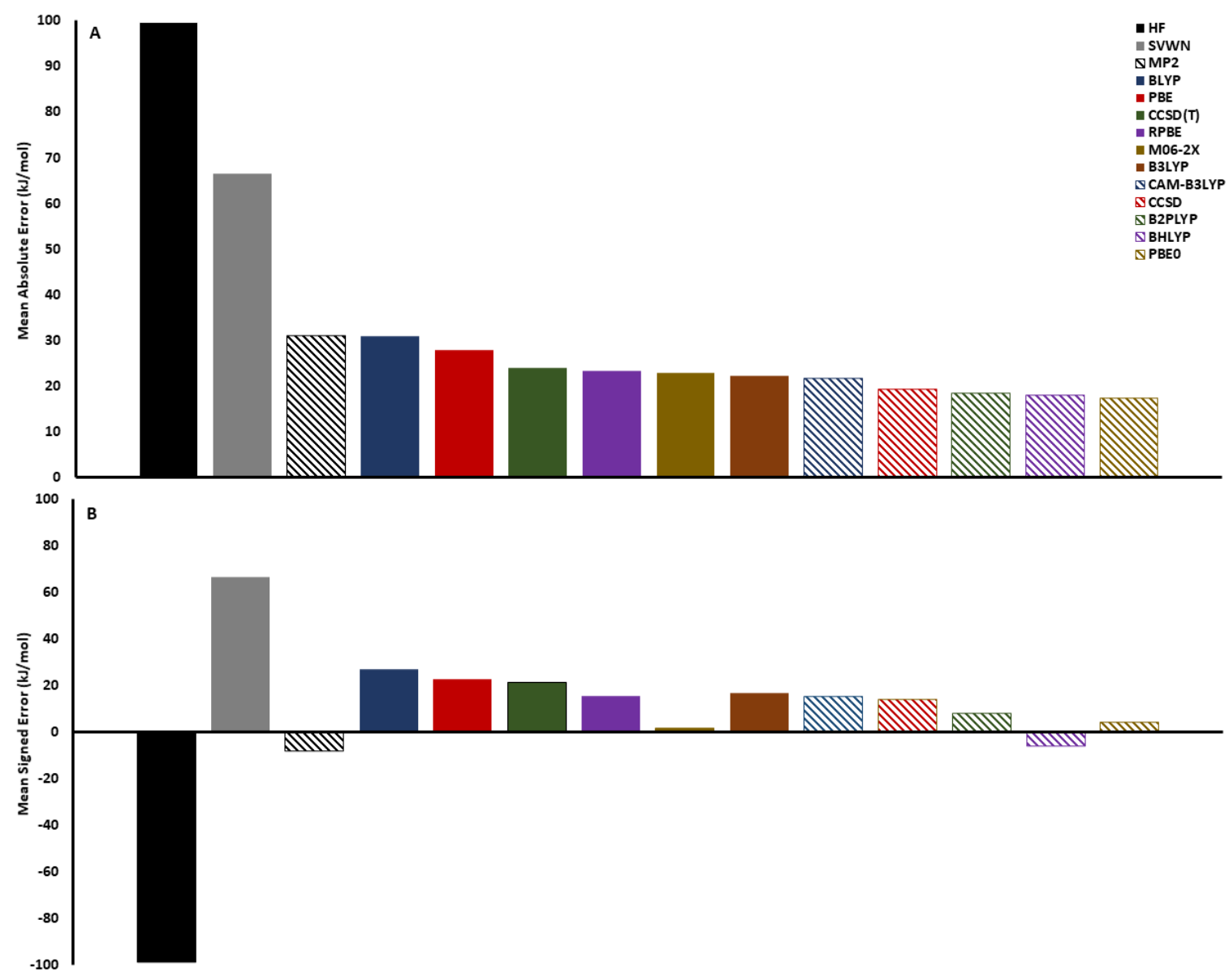

Figure 1. A) Mean unsigned errors of all methods vs. the 50 experimental $M-H$ and $\mathrm{M}^{+}-\mathrm{H}$ bonds. B) The corresponding mean signed errors.

The most accurate functionals are the PBE0 and BHLYP global hybrids and the B2PLYP double hybrid with 25, 50 and 53\% HF exchange, having MAEs of 17.3, 18.2 and $18.6 \mathrm{~kJ} / \mathrm{mol}$, respectively. CAM-B3LYP and B3LYP with 19-65 and 20\% HF exchange also perform well, with MAEs of 21.8 and $22.2 \mathrm{~kJ}$. The gradient-corrected non-hybrid functionals with 0\% HF exchange (BLYP and PBE) perform quite poorly (MAE of 30.9 and $28.0 \mathrm{~kJ} / \mathrm{mol}$ ). Thus, higher HF exchange up to 50\% describes the M-H bond more accurately. M06-2X with 54\% HF exchange and RPBE with 0\% HF exchange have MAEs of $22.9 \mathrm{~kJ} / \mathrm{mol}$ and 23.4 $\mathrm{kJ} / \mathrm{mol}$, respectively, making M06-2X and RPBE comparable in performance to B3LYP. The performance of M06-2X relates to the fact that it is heavily parametrized mainly for nonmetals ${ }^{34}$, and thus it is less useful for providing physical insight in this particular study, 
although its performance is decent. The relatively good performance of RPBE compared to e.g. PBE also having 0\% HF exchange relates to the fact that RPBE by design binds more weakly than PBE in an attempt to improve the description of chemisorption on metal surfaces ${ }^{39}$. As expected, the LDA functional SVWN produces very large overbinding and a correspondingly large MAE of $66.6 \mathrm{~kJ} / \mathrm{mol}$.

The MSEs in Figure 1B describe the systematic tendency towards overbinding or underbinding. It importantly shows that for the functionals selected here, we can approach a maximal systematic accuracy because HF-exchange reduces the over-binding tendency to approximately $0 \mathrm{~kJ} / \mathrm{mol}$ towards the right. Thus for example, the BLYP functional has an MSE of $26.6 \mathrm{~kJ} / \mathrm{mol}$ while the BHLYP functional has an MSE of $-6.0 \mathrm{~kJ} / \mathrm{mol}$. This trend in systematic errors is fundamentally different from that of other M-L bonds, which are adequately described by $0-20 \%$ HF exchange, depending on the nature of $\mathrm{L}^{15,17,55}$.

This major difference is important to the errors of a theoretical study of any catalytic reaction involving both $\mathrm{M}-\mathrm{H}$ and $\mathrm{M}-\mathrm{L}$ bonds. To illustrate this more clearly, let us take as example transition-metal catalyzed ammonia synthesis. M-O and M-N bonds of transition metals are adequately described by $10-20 \%$ HF exchange ${ }^{10,15,17}$, and for some late-transition metal atoms such as gold ${ }^{14}$, and for halide ligands ${ }^{10}$, the HF requirements are even smaller. Any attempt to correctly estimate the thermochemistry of the catalytic cycle requires balancing the errors of the hydride and nitride states (M-H and $\mathrm{M}-\mathrm{N})$, as well as potentially the adsorbed $\mathrm{H}-\mathrm{H}$ and N-N states, which are however more similar for different metals and thus produce smaller errors in the trend chemistry of different $\mathrm{M}$, as crucially affecting e.g. volcano plots. Using a functional such as PBE or RPBE will favor a more appropriate description of the more statically correlated M-N bonds at the expense of error-prone M-H bonds. Alternatively, a higher HF exchange such as in BHLYP would provide a good description of the M-H bonds 
as shown in Figure 1, but a worse description of $\mathrm{M}-\mathrm{N}$ bonds ${ }^{16}$. Thus, when studying such a catalytic process, DFT is fundamentally unbalanced because of the distinct requirements of the different electronic states (M-H, M-N, N-N etc.). For the light-atom bonds (O-O, N-N, C-O) non-hybrid GGAs such as PBE dramatically overbind from 100 to $40 \mathrm{~kJ} / \mathrm{mol}$, decreasing with effective nuclear charge. This unbalance can be partly reduced by systematic error cancellation, since the error made in the M-H states is potentially similar for different M. Hybrid functionals with a modest amount of HF exchange such as PBE0, range-separated hybrids (CAM-B3LYP) and double hybrids (B2PLYP) have the smallest systematic errors (Figure 1B) and also performed well in L-L and M-L benchmarks ${ }^{10,56}$, making them the best choices for these problems.

The performance of $\operatorname{CSSD}(\mathrm{T})$ can be seen to be lower than most of the studied density functionals, giving a MAE of $24.1 \mathrm{~kJ} / \mathrm{mol}$. CCSD(T) also performs worse than CCSD with a MAE of $19.5 \mathrm{~kJ} / \mathrm{mol}$. This difference in MAE is larger than the uncertainty in MAE due to experimental data variations as shown from our sensitivity analysis in the methods section. Accordingly, it implies a lack of a treatment of static correlation in the post-HF methods, which is inherently but indirectly treated adequately by DFT. HF with neither static nor dynamic correlation fails massively. MP2 which adds dynamic correlation performs much better, but misses static correlation that makes it still the third-worst method. CCSD and CCSD(T) greatly improves upon this description. The missing static correlation in the coupled-cluster description breaks the expected behavior of improvement from CCSD to CCSD(T), which is very unusual.

Origins of the metal hydride problem: Electron density effects. Recent focus on the accuracy of DFT has emphasized both the two fundamental dimensions of DFT, the density and energy, rather than just the latter. ${ }^{57,58}$ Any choice of density error is unfortunately 
somewhat arbitrary: Results are very dependent on how the density error is measured, and thus these errors only make sense in relation to the energy of the very same systems ${ }^{57}$.

As suggested by Burke, ${ }^{59}$ the error in a DFT calculation can be divided into a densitydriven error and functional-driven error (the error in energy due to the functional itself). Calculations having a large density-driven error are classified as abnormal. ${ }^{59}$ Such a metric that uses the energy effect of the density error as a single number is the only reasonable way to avoid arbitrary density error estimates central to the current debate on DFT. ${ }^{49,57}$ Unfortunately, this definition requires knowledge of the exact or near-exact energy and density, which is elusive for most chemical systems of interest. Instead, our solution ${ }^{49}$ to the energy-density conundrum is go to the root of the problem, i.e. the variational Hohenberg-Kohn theorem, and perform a sensitivity analysis to simply measure the effect on energy of a reasonable variation in electron density ${ }^{49}$. For this purpose, one should use a set of trial densities computed using different functionals, preferably from different rungs of Jacob’s ladder ${ }^{60}$. Our protocol to do this that can involve any number of density functionals used to generate trial densities and then the computation of the energy on all these densities to quantify energy sensitivity to density ${ }^{49}$. For a four-functional trial set (used as illustration to cover the first four rungs), this becomes a somewhat tedious calculation, and Sim et al. ${ }^{50}$ accordingly suggested a simpler $S\left(\widetilde{E}_{X C}\right)$ metric using only the SVWN and HF densities, which is much more sensitive than a pure DFT metric, because the HF density is so distinct. ${ }^{50}$ 


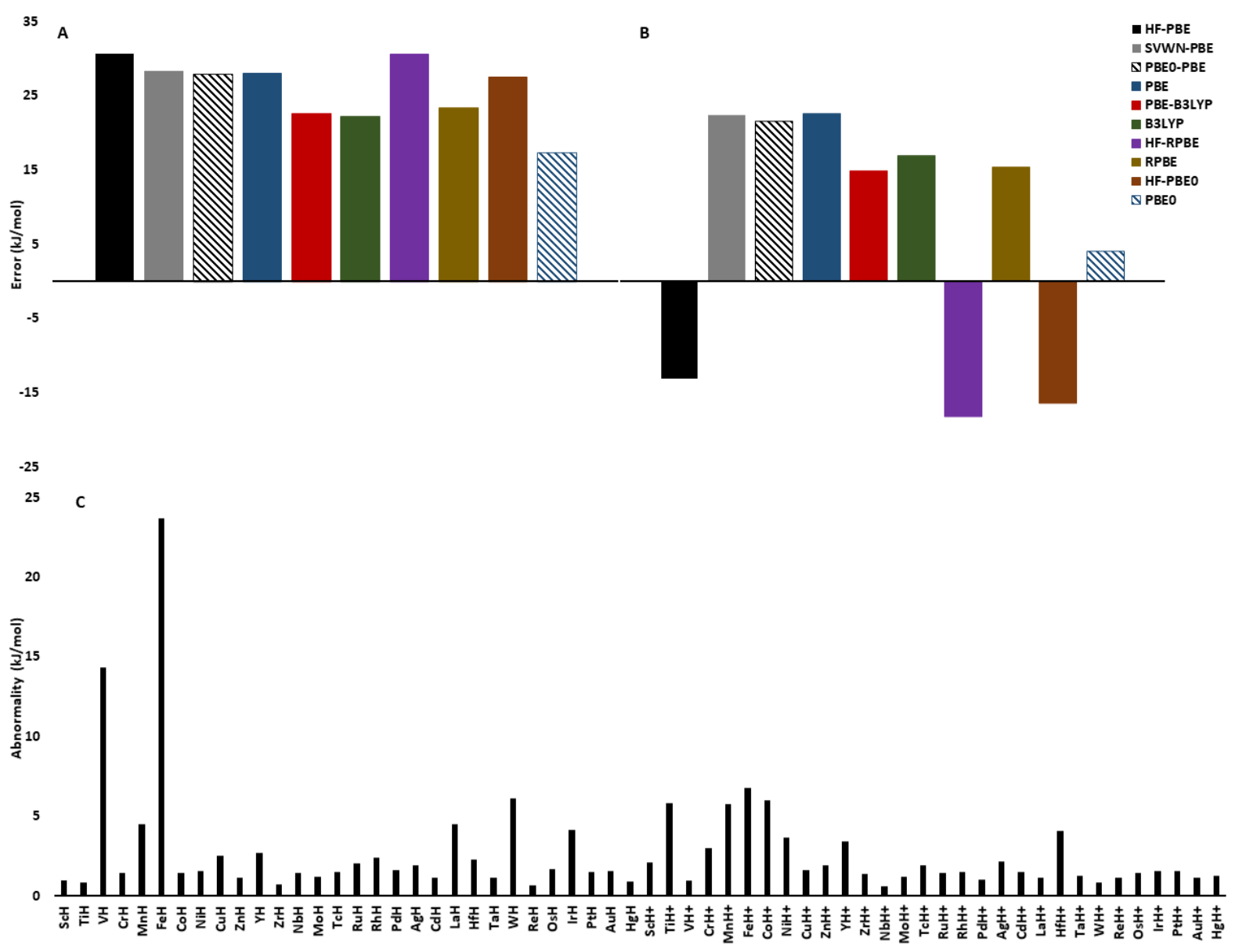

Figure 2. A) Mean absolute errors for various versions of HF-DFT and their corresponding pure functionals, vs. 50 experimental M-H BDEs. B) Corresponding mean signed errors. C) Electronic abnormality = difference in the PBE energy evaluated at the SVWN and PBE0 densities.

Figure 2A and 2B summarize the performance of the HF-DFT, LDA-DFT and PBEB3LYP methods (the latter representing a B3LYP energy evaluated on a PBE density). The performance is substantially worse than for the corresponding self-consistent treatments, with MAEs of 27.5-30.7 kJ/mol. We conclude from this that there is a large density-derived error in the systems, larger than commonly the case ${ }^{59}$, but that HF-DFT is not a solution to this problem, because the uncorrelated HF density makes performance markedly worse despite reducing the SIE (see below). 
The $S\left(\widetilde{E}_{X C}\right)$ metric suggested by Sim et al. ${ }^{50}$ was calculated for all 60 systems and can be seen in Table S32. Many values are very large, and they average to $36 \mathrm{~kJ} / \mathrm{mol}$ (standard deviation: $33 \mathrm{~kJ} / \mathrm{mol}$ ) for all 60 systems. Thus, almost all systems would be classified as abnormal and this precludes the sensitivity needed for our analysis. More importantly, the numbers are very affected by the pathology of the HF framework, which we consider unreasonable from a pure DFT perspective. In fact, although HF-DFT was suggested to improve results for abnormal systems, ${ }^{50}$ as seen from the MAEs in Figure 2A it does the contrary due to the adverse impact of the HF density. The reason is that for statically correlated systems residing in the d-transition groups, HF is so pathological that it is unacceptable to use HF-DFT, and the metric was only tested on s- and p-group systems. The systematic errors in Figure 2B show that the HF density overcompensates the reduction in binding by changing the sign of the method even though the same functional is used for the energy.

Figure 2 also shows the MAE for all 50 calculations with self-consistent B3LYP vs. the 50 calculations with B3LYP on PBE densities (PBE-B3LYP). As seen, using a PBE density as basis for calculating the energy with the substantially different functional B3LYP does not influence the accuracy of the calculation to a significant extent, consistent with our previous suggestion that PBE-DFT (where DFT is the functional used for the energy, and PBE is the density) is a powerful fast method of similar quality as the DFT method itself ${ }^{49}$. The same conclusion can be drawn even using a SVWN density (SVWN-PBE). This analysis indicates that the electron density, once correlated by DFT, which indirectly accounts for static correlation, does not influence the accuracy for metal hydrides to any large extent.

We conclude that density sensitivity analysis ${ }^{49}$ is a useful tool in the analysis of DFT calculations. However, despite the non-empirical appeal of local DFT and HF, the simplified metric suggested by Sim et al. ${ }^{50}$ produces too large and insensitive estimates of abnormality 
because the HF density is so pathological. Rather, we revert to a pure DFT metric of density sensitivity as originally suggested ${ }^{49}$, but using the difference in PBE energy computed on an SVWN and a PBE0 density, i.e. E(SVWN-PBE)-E(PBE0-PBE). This abnormality metric contains DFT correlation for both densities but at the local and hybrid level, to ensure a reasonable density variation of the type expected in real systems without suffering the pathology of pure HF densities. This measure of abnormality is shown in Figure 2C and directly quantifies the sensitivity of the computed BDE to variations in electron density at the DFT-correlated level. The two most abnormal systems are, not surprisingly, the first-row 3d block FeH and $\mathrm{MnH}$ systems which are in the middle of the first 3d transition period and thus particularly subject to close-lying d configurations, with a consequential larger effect of using a density with some vs. no HF included. For most other systems, the metric gives $1-5 \mathrm{~kJ} / \mathrm{mol}$, in the order of chemical accuracy, which is a suitable threshold for separating abnormal systems ${ }^{49}$, and thus it effectively separates the abnormal systems as required.

SIE contributions to the description of the metal hydrogen bond. The pure $\mathrm{M}-\mathrm{H}$ bonds are unusual, not so much by the fact that HF is pathological, which is true for many statically correlated M-L bonds ${ }^{61,62}$, but because DFT still requires a large component of the HF exchange to perform accurately (this changes with the modulation from larger coordination number, see below). DFT applied to transition metal chemistry is characterized by a trade-off between the need for electron correlation and the problem of self interaction error (SIE), with these two features commonly in conflict. ${ }^{7,51,63,64}$ SIE represents the failure of density functionals when the exchange-correlation treatment abandons the exact cancellation of the exchange and Coulomb integrals for a given electron (the self energy). The problem manifests in particular for diffuse odd-electron densities where the electrons are artificially delocalized. 
The extent of SIE in many-electron systems is currently a matter of debate $e^{51,52,63-65}$. The archetypical pathological case of SIE is the dissociation curve of $\mathrm{H}_{2}{ }^{+}$, where DFT produces artificially and increasingly stable states along the dissociation potential energy curve, as illustrated for convenience with a few examples in Figure 3A (this curve has been shown for select functionals in many papers previously). ${ }^{65,66}$ To estimate the importance of SIE in the metal hydrides, we computed the SIE using Equation 4 as suggested by Zhang \& Yang ${ }^{51}$ :

$$
S I E=J\left[\rho_{\mathrm{q}}\right]+\mathrm{E}_{\mathrm{xc}}\left[\rho_{\mathrm{q}}\right]
$$

Here $\mathrm{J}\left[\rho_{\mathrm{q}}\right]$ is the classical electron-electron repulsion for density $\rho_{q}$ and $\mathrm{E}_{\mathrm{xc}}\left[\rho_{\mathrm{q}}\right]$ is the exchange-correlation energy for density $\rho_{q}$. The SIE was calculated for the $\mathrm{H}_{2}{ }^{+}$system with the two $\mathrm{H}$ atoms separated by $10 \AA$ where we know the system behaves pathologically (values in Table S33). Please note that for B2PLYP the pure-DFT-derived SIE is computed for comparison to other methods, ignoring the modifying influence of the MP2 correlation energy.

Figure 3B shows the correlation between $|\mathrm{SIE}|$ and the MAE of the GGA and hybrid functionals, where gradient-corrected exchange-correlation is expected to produce SIE (the range-separated CAM-B3LYP does not have a well-defined SIE as the HF exchange is variable and was not included). The MAEs of the methods scale with the SIE with $\mathrm{R}^{2}=0.70$. RPBE deviates from this trend because it was reparametrized to increase performance for M-L bonding ${ }^{39}$ but without reducing the SIE of PBE. Removing RPBE increases $\mathrm{R}^{2}$ to 0.84 , further strengthening this relationship. We conclude that functionals with a lower SIE tend to describe the $\mathrm{M}-\mathrm{H}$ bond more accurately. Thus, the pathology of the $\mathrm{M}-\mathrm{H}$ bonds is largely due to SIE, and partly to correlation of $3 \mathrm{~d} / 4 \mathrm{~d} / 5 \mathrm{~d}$ and 4 s configurations. Figure $3 \mathrm{C}$ shows the relationship between the SIE and the amount of HF exchange, which as expected is linear with $\mathrm{R}^{2}=0.998$. 


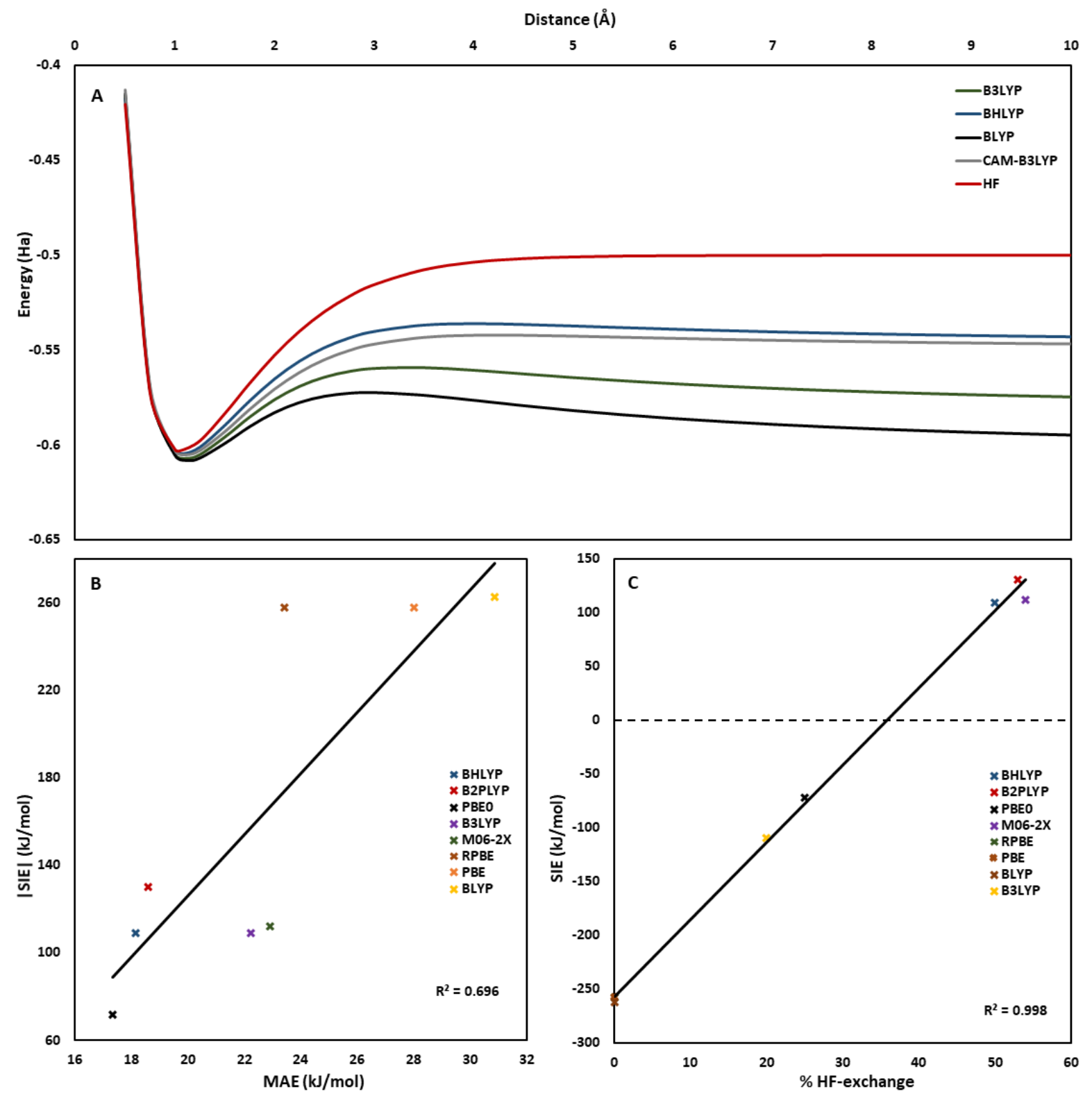

Figure 3. A) Energy of $\mathrm{H}_{2}{ }^{+}$as a function of distance for selected functionals. B) Correlation between the absolute value of SIE (Equation 4) and the MAE for all 50 metal hydrides. C) Linear relationship between SIE and the amount of HF-exchange in GGA functional. 

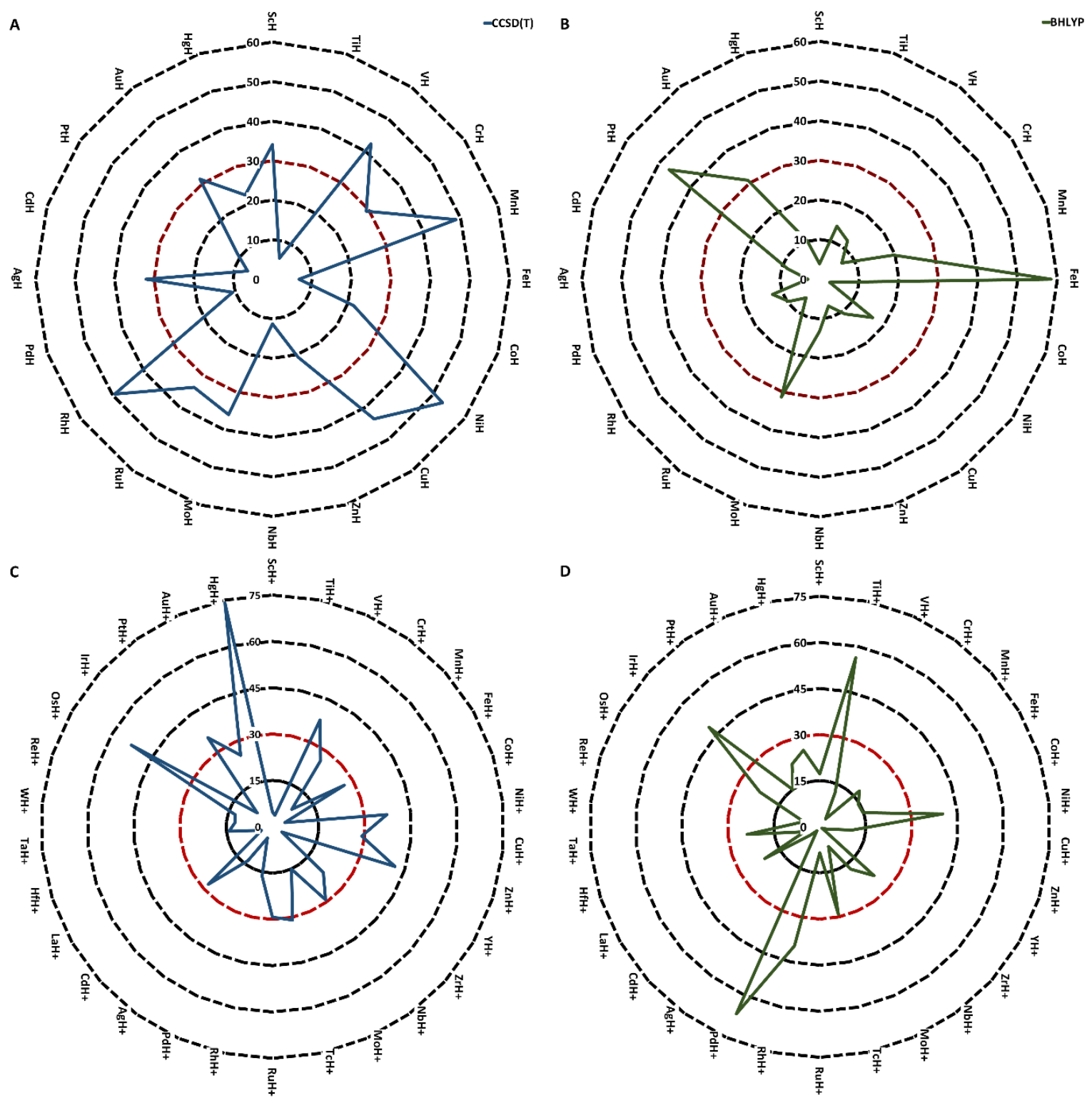

D

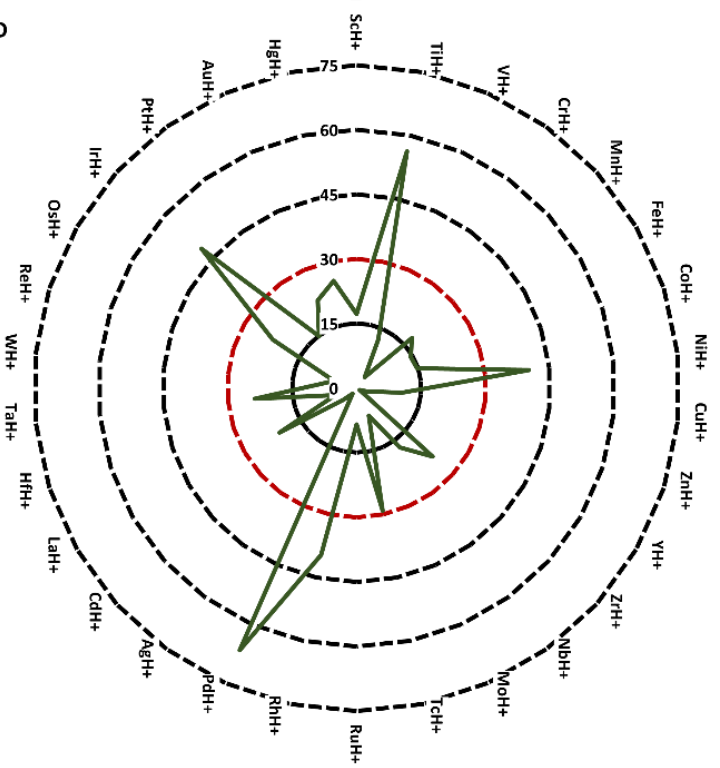

Figure 4. A) Absolute error of M-H systems for CCSD(T). B) Absolute error of M-H systems

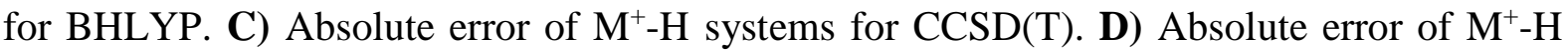
systems for BHLYP. A threshold of $30 \mathrm{~kJ} / \mathrm{mol}$ (red color) is used to identify pathological cases.

Figure 3B and 3C in combination explain why the pure M-H bonds impose particularly high HF exchange requirements. The SIE is reduced significantly going from $0 \%$ HF-exchange to $20 \%$ in B3LYP. From B3LYP to BHLYP with 50\% HF-exchange it becomes positive. Accordingly, we immediately identify an optimal value of HF exchange of 35\% as producing 
zero SIE and minimal MAE for the M-H bonds in the limit of low coordination number (=1). Consistent with this interpolation, PBE0 (25\%) and BHLYP (50\%) are of similar accuracy but on opposite sides of the linear trend curve in Figure 3B. The reduced amount of SIE for functionals with more HF-exchange also explains why B2PLYP and CAM-B3LYP produce low MAEs for the M-H bonds, as they enable more of the SIE-free HF-exchange without loss of accuracy (Table 1).

Difficult systems. To understand the system-dependencies and accuracy bottlenecks more completely, we divided errors into system type. Tables S34-S40 show the errors for the density functionals divided into atomic configuration being either $s^{0}, s^{1}$, or $s^{2}$, and into d-block (3d, 4d, 5d) and period group number. It emerges from Table S34 that DFT has particular problems with the $s^{1}$ configurations, whereas other system categorizations produce similar performance across system types. The $\mathrm{s}^{1}$ configuration, due to the diffuse nature of this single, outermost electron, is a likely contributor to SIE in the atomic and ionic systems, supporting the analysis above.

Figure 4 shows the absolute errors for all systems computed using $\operatorname{CCSD}(\mathrm{T})$ and BHLYP, as a representative well-performing functional with a large amount of HF exchange. A pathological case is defined here as a system where the absolute error compared to experiment is $>30 \mathrm{~kJ} / \mathrm{mol}$ (indicated by the red circles in Figure 4). For CCSD(T) these systems are $\mathrm{ScH}, \mathrm{VH}, \mathrm{MnH}, \mathrm{NiH}, \mathrm{CuH}, \mathrm{MoH}, \mathrm{RuH}, \mathrm{RhH}, \mathrm{AgH}, \mathrm{AuH}, \mathrm{VH}^{+}, \mathrm{NiH}^{+}, \mathrm{ZnH}^{+}, \mathrm{TcH}^{+}, \mathrm{OsH}^{+}$, $\mathrm{PtH}^{+}$, and $\mathrm{HgH}^{+}$. For BHLYP the systems are: $\mathrm{FeH}, \mathrm{MoH}, \mathrm{PtH}, \mathrm{AuH}, \mathrm{TiH}^{+}, \mathrm{NiH}^{+}, \mathrm{RhH}^{+}, \mathrm{PdH}^{+}$, and $\mathrm{IrH}^{+}$. BHLYP has nearly half as many pathological systems (9) as $\operatorname{CCSD(T)~(17).~Figure~}$ 4 illustrates the important trade-off between static correlation and SIE that particularly haunts the metal hydrides. We are operating with large basis sets with small errors for these problems $^{10}$; the calculations adequately reflect the thermochemical observable BDE, and 
relativistic effects are well-described as seen from the uniform performance across all three periods of the d-block; this comparative test is our preferred assessment of the adequacy of relativistic corrections as these cannot be measured experimentally.

If the system is statically correlated, $\operatorname{CCSD}(T)$ is expected to have difficulties describing it. The D1 diagnostic of CCSD(T) has been suggested as a proxy of multi-reference character. ${ }^{67}$ Of the 17 systems that are pathological for $\operatorname{CCSD}(\mathrm{T}), \mathrm{ScH}$ and VH have D1 values above 0.15 and many others have values higher than 0.05 . As also established by Jiang et al. it does not exclude other systems from also having multi-reference character as the metric is sensitive but not sufficient. The six systems $\left(\mathrm{FeH}, \mathrm{PtH}, \mathrm{TiH}^{+}, \mathrm{RhH}^{+}, \mathrm{PdH}^{+}, \mathrm{IrH}^{+}\right)$that are pathological only for BHLYP but not for $\operatorname{CCSD}(\mathrm{T})$ are likely suffering from an incomplete description of the dynamic correlation or SIE of BHLYP, since CCSD(T) describes them well. We note the adjacent position of Pd, Pt, Rh, and Ir in the periodic table in this regard, all being important metals in terms of catalyzed $\mathrm{M}-\mathrm{H}$ bond breaking and formation.

Study of coordinatively saturated complexes. In the above analysis, we showed that HF requirements for pure M-H bonds are much larger (on average 35\%) than for typical M-L diatomics and for normal coordinatively saturated complexes, which are typically 020\%. ${ }^{7,10,15,17}$ These systems are relevant as the pure unmodified $\mathrm{M}-\mathrm{H}$ bonds and as the limit of low coordination number. In catalytic reactions the coordination number typically changes. In order to also test the limit of high coordination number which is more commonly encountered in real reactions, we performed a new study of 19 coordinatively saturated complexes with experimentally known BDEs for the M-H bonds, and computed their M-H BDEs as described in the Methods section. These 19 systems in their H-bound form are shown in Figure 5. The main results are summarized in Figure 6, with BDEs given in the Supporting Information, Tables S29-S30, and the HOMO-LUMO gaps in Tables S41-S42. 

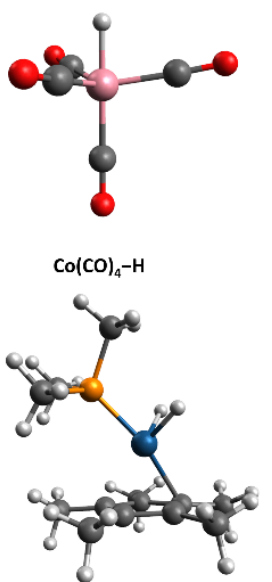

$\operatorname{Ir}(\mathrm{H})\left(\eta^{5}-\mathrm{C}_{5} \mathrm{Me}_{5}\right)\left(\mathrm{PMe}_{3}\right)-\mathrm{H}$

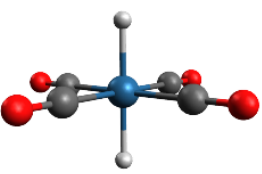

$\mathrm{Os}(\mathrm{H})(\mathrm{CO})_{4}-\mathrm{H}$

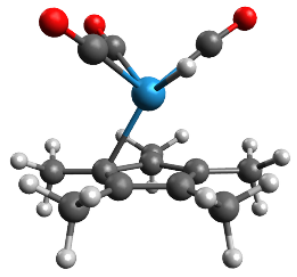

$\mathrm{W}(\mathrm{CO})_{3}\left(\eta^{5}-\mathrm{C}_{5} \mathrm{H}_{5}\right)-\mathrm{H}$

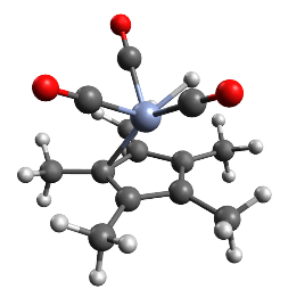

$\left(\eta^{5}-\mathrm{C}_{5} \mathrm{H}_{5}\right) \mathrm{Cr}(\mathrm{CO})_{3}-\mathrm{H}$

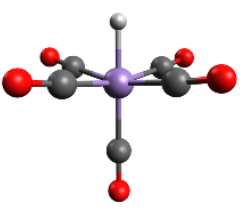

(CO) $)_{5} \mathrm{Mn}-\mathrm{H}$
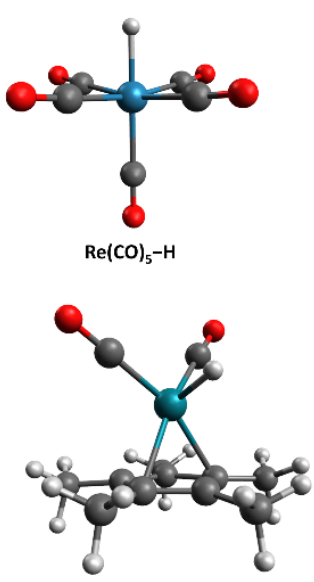

$\left(n^{5}-\mathrm{C}_{5} \mathrm{H}_{5}\right)(\mathrm{CO})_{2} \mathrm{Rh}^{+}-\mathrm{H}$

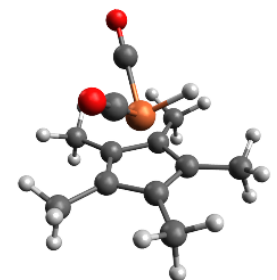

$\left(\eta^{5}-\mathrm{C}_{5} \mathrm{H}_{5}\right)(\mathrm{CO})_{2} \mathrm{Fe}-\mathrm{H}$

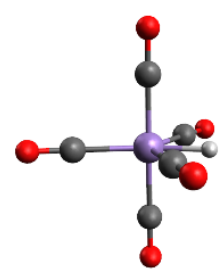

(CO) $\mathrm{Mn}^{+-}-\mathrm{H}$
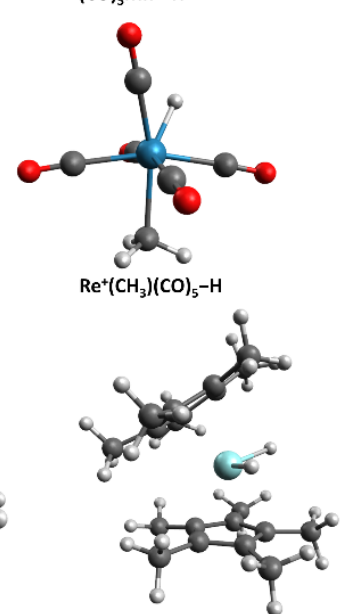

$\mathrm{Zr}(\mathrm{H})\left(\mathrm{n}^{5}-\mathrm{C}_{5} \mathrm{Me}_{5}\right)_{2}-\mathrm{H}$
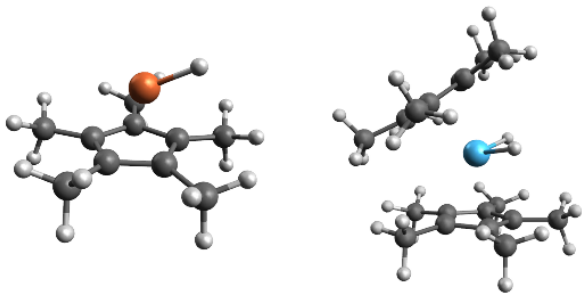

$H f(H)\left(\eta^{5}-C_{5} M_{5}\right)_{2}-H$

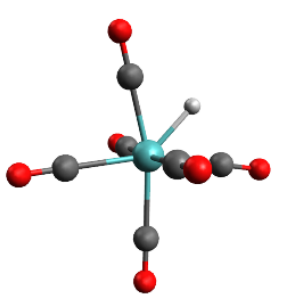

$(\mathrm{CO})_{6} \mathrm{Mo}^{+}-\mathrm{H}$
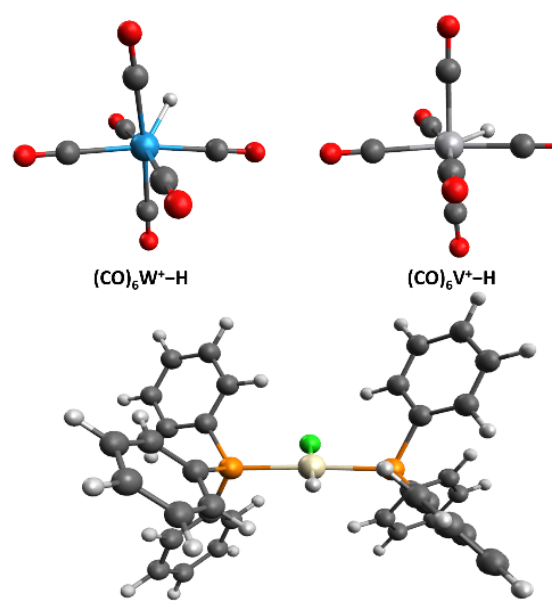

Trans-Pt( $\left(\mathrm{PPh}_{3}\right)_{2}(\mathrm{Cl})-\mathrm{H}$

Figure 5. The 19 saturated coordination complexes studied in this work.

As seen from the MAEs in Figure 6A and the MSEs in Figure 6B, the methods are still challenged in describing the M-H bonds, but a remarkable change towards very low HF requirements is seen for high coordination number. Thus, non-hybrid GGA functionals perform very well for the saturated complexes although they fail for the diatomics. Reversely, high-HF hybrids such as PBE0 and BHLYP fail substantially for high coordination number. Our finding that HF requirements change dramatically with coordination number can be explained from the changed relative importance of SIE vs. correlation effects of the electron-dense states at high coordination number. The balance of errors due to SIE and correlation effects of the d-shells is now shifted toward the latter, such that the HF treatment becomes further pathological. 

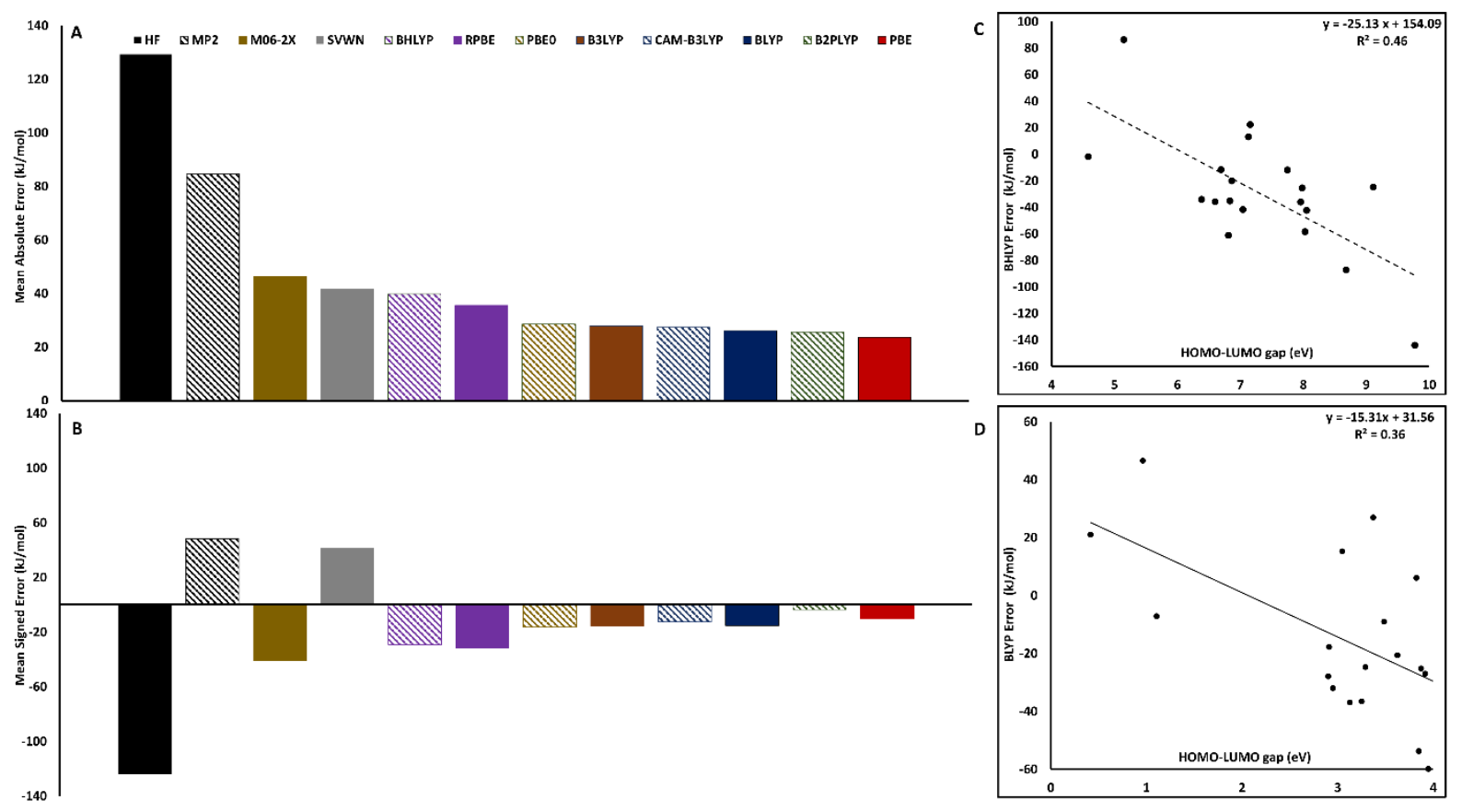

Figure 6. A) Mean absolute errors (in $\mathrm{kJ} / \mathrm{mol}$ ) of ten density functionals, HF, and MP2 for computing the M-H BDE of 19 coordinatively saturated complexes shown in Figure 5. B) The corresponding mean signed errors. C) Relationship between the HOMO-LUMO gap (in eV) and the error in M-H BDE, computed with BHLYP with 50\% HF exchange. D) Same as C, but for BLYP with 0\% HF exchange.

We hypothesized that the failure of DFT due to this balance can be reflected in the HOMO-LUMO gap because it is a proxy of the density of states. Figure 6C and 6D show the relationship between the HOMO-LUMO gap and the error in BHLYP and BLYP vs. experimental BDE for the 19 saturated complexes, as representative extremes of high and low HF exchange fractions ( $50 \%$ and $0 \%$ ). In both cases, despite the expectedly large scatter, the relationship is statistically significant at the 95\% confidence level (P-values 0.0013 and 0.0071 ) and suggests that the functionals overbind for small gaps (high density of states) and underbind for large gaps (low density of states). 
Importantly, we find that B2PLYP and CAM-B3LYP still performs well for the saturated complexes (Figure 6A/B). Thus, while DFT is fundamentally unbalanced in its HF requirements as a function of coordination number, which typically changes during catalytic reactions, these two functionals remedy this problem and are the only transferable (or “universal”) functionals for treating $\mathrm{M}-\mathrm{H}$ bonds across the coordination number range. This is well in line with the analysis above that these methods balance the HF exchange requirements with the electronic diversity and density of states of the two regimes of coordination number, where low coordination number errors are dominated by SIE that make the HF picture more valid and high coordination numbers by correlation effects that make the HF picture less valid.

Why metal hydrogen bonds are particularly SIE-prone. The failure of HF and modest performance of CCSD(T) on one hand, and the better but variable performance of DFT on the other hand show that metal hydrides are unique in their combined requirements for a treatment of static correlation and SIE. This is relatively easy to understand: The metals have low electronegativity and therefore enforce a hydride-like state upon the hydrogen atom in the system. Because the proton charge is only 1 , the hydride state is extremely diffuse. In combination with a bonding that involves unpaired electrons allocated to a diffuse hydrogen density, this creates SIE that is unprecedented in any equilibrium geometry system that we are aware of, since the ratio of electrons per effective nuclear charge is probably the maximally attainable in these systems. We thus conclude that they represent a prominent example of many-electron molecules with major SIE already in their ground state geometries $\left(\mathrm{Ne}_{2}{ }^{+}\right.$and other odd-electron molecules have SIE upon dissociation but not at equilibrium). Accordingly, the metal hydrides are not only very important catalytically, they are also very special electronically. 
Trend chemistry for metal hydrogen bonds. The trend chemistry, which is ultimately the most important aspect of performance in many applications e.g. for comparing different metal catalysts, is summarized in Figures S3-S22. Following consistently the conclusions from the analysis above, we find that all methods that use the HF density directly (HF-DFT) show very poor trend accuracy compared to the pure DFT methods (typical $\mathrm{R}^{2}=0.79-0.88$ ) and $\operatorname{CCSD} / \operatorname{CCSD}(\mathrm{T})$, which notably show the best trend accuracy $\left(\mathrm{R}^{2}=0.90\right)$. The generally high trend accuracy of DFT lends promise to possible cancellation of systematic errors that may reduce some of the errors identified in this analysis when using DFT comparatively, although this requires specific documentation in any actual application of computational catalysis involving formation or breaking of $\mathrm{M}-\mathrm{H}$ bonds.

\section{Conclusions}

Transition metal hydrides, despite their enormous importance to many catalytic processes, pose a challenge not only to DFT but also to correlated post-HF wave function methods that use a single Slater determinant as their reference state. To understand when, why, and by how much DFT fails to describe the M-H bond, we explored all $30 \mathrm{M}-\mathrm{H}$ and $30 \mathrm{M}-\mathrm{H}^{+}$systems of the 3d, $4 \mathrm{~d}$, and $5 \mathrm{~d}$ metals. 50 of these 60 systems have known BDEs that we can conveniently use as benchmark. We design various tests and metrics for SIE and abnormality that help us dissect the physical origins of the failure to describe the $\mathrm{M}-\mathrm{H}$ bonds. Since coordination numbers vary substantially in real catalytic reactions, we also explored the limit of high coordination number using a data set of 19 coordinatively saturated complexes with known M-H BDEs.

The ten main findings arising from this work are: 1) the HF method fails to describe the M-H bond due to its lack of correlation; 2) accordingly, post-HF methods such as MP2 and even CCSD(T) perform worse than many density functionals; 3) for the pure bonds in the 
diatomic molecules, DFT requires much more HF exchange ( 35\%) to describe M-H bonds than other metal ligand bonds (0-20\%); 4) we design a test to determine if self-interaction error (SIE) is important by correlating DFT errors against a one-electron SIE metric; 5) we show that SIE correlates directly with the DFT errors and thus causes most of the problem; 6) HFDFT cannot handle these systems because the HF method is too pathological already at the density level; 7) instead, we define and apply a simple metric of electronic abnormality as the difference in PBE energy computed at the self-consistent PBE0 and SVWN densities; this metric gives appropriate spread and effectively captures density-derived errors; $\mathrm{MnH}$ and $\mathrm{FeH}$ are particularly abnormal, due to their close-lying d-configurations in the middle of the d-block; 8) the low electronegativity of the metal enforces a diffuse hydride-like electron density, which make the metal hydrides primary examples of many-electron systems exhibiting SIE already at equilibrium geometries; 9) in the coordinatively saturated $\mathrm{ML}_{n} \mathrm{H}$ systems, much less $\mathrm{HF}$ exchange is required, i.e. the HF exchange requirements vary drastically with coordination number. Accordingly, DFT is unbalanced when studying any catalytic process involving both M-H and M-L bonds (L being a non-hydrogen-ligand) with changing coordination numbers; 10) the range-separated and double-hybrid functionals CAM-B3LYP and B2PLYP alone perform well for both M-H and M-L systems, and in both limits of low and high coordination number, and at least as well as $\operatorname{CCSD}(\mathrm{T})$, because they balance the HF exchange requirements across the electronic diversity caused by the coordination number. Since these methods are transferable between low and high coordination number and M-L type, they lend hope to the possibility of a balanced (i.e. universal) application of DFT to catalysis, which we show is impossible with standard global hybrids or GGA functionals. 


\section{Acknowledgements}

We acknowledge the use of the Steno Cluster at DTU Chemistry, originally funded by The Danish Council for Independent Research | Natural sciences (FNU).

\section{Supporting Information available}

The Supporting information file contains 42 tables and 22 figures with the experimental data for the $20 \mathrm{M}-\mathrm{H}$ systems and $30 \mathrm{M}^{+}-\mathrm{H}$ systems, and for the 19 coordinatively saturated $\mathrm{ML}_{n} \mathrm{H}$ systems, computed electronic energies with all functionals, computed BDEs, mean absolute errors and signed errors divided into groups, and linear correlation plots. This material is available free of charge via the Internet at http://pubs.acs.org. 


\section{References}

(1) McGrady, G. S.; Guilera, G. The Multifarious World of Transition Metal Hydrides. Chem. Soc. Rev. 2003, 32 (6), 383-392.

(2) Liu, L.; Corma, A. Metal Catalysts for Heterogeneous Catalysis: From Single Atoms to Nanoclusters and Nanoparticles. Chem. Rev. 2018, 118 (10), 4981-5079.

(3) Hinnemann, B.; Moses, P. G.; Bonde, J.; Jørgensen, K. P.; Nielsen, J. H.; Horch, S.; Chorkendorff, I.; Nørskov, J. K. Biomimetic Hydrogen Evolution: MoS2 Nanoparticles as Catalyst for Hydrogen Evolution. J. Am. Chem. Soc. 2005, 127 (15), 5308-5309.

(4) Ferrin, P.; Kandoi, S.; Nilekar, A. U.; Mavrikakis, M. Hydrogen Adsorption, Absorption and Diffusion on and in Transition Metal Surfaces: A DFT Study. Surf. Sci. 2012, 606 (7-8), 679-689.

(5) Kohn, W.; Becke, A. D.; Parr, R. G. Density Functional Theory of Electronic Structure. J. Phys. Chem. 1996, 100 (31), 12974-12980.

(6) Mardirossian, N.; Head-Gordon, M. Thirty Years of Density Functional Theory in Computational Chemistry: An Overview and Extensive Assessment of 200 Density Functionals. Molecular Physics. Taylor \& Francis 2017, pp 2315-2372.

(7) Kepp, K. P. Consistent Descriptions of Metal-ligand Bonds and Spin-Crossover in Inorganic Chemistry. Coord. Chem. Rev. 2013, 257 (1), 196-209.

(8) Ziegler, T.; Li, J. Bond Energies for Cationic Bare Metal Hydrides of the First Transition Series: A Challenge to Density Functional Theory. Can. J. Chem. 1994, 72 (3), 783789. 
(9) Zhang, W.; Truhlar, D. G.; Tang, M. Tests of Exchange-Correlation Functional Approximations against Reliable Experimental Data for Average Bond Energies of 3d Transition Metal Compounds. J. Chem. Theory Comput. 2013, 9 (9), 3965-3977.

(10) Moltved, K. A.; Kepp, K. P. Chemical Bond Energies of 3d Transition Metals Studied by Density Functional Theory. J. Chem. Theory Comput. 2018, 14, 3479-3492.

(11) Jacobsen, C. J. H.; Dahl, S.; Clausen, B. S.; Bahn, S.; Logadottir, A.; Nørskov, J. K. Catalyst Design by Interpolation in the Periodic Table: Bimetallic Ammonia Synthesis Catalysts. J. Am. Chem. Soc. 2001, 123 (34), 8404-8405.

(12) Hammer, B.; Nørskov, J. K. Theoretical Surface Science and Catalysis-calculations and Concepts. In Impact of Surface Science on Catalysis; Academic Press, 2000; Vol. 45, pp 71-129.

(13) Wellendorff, J.; Silbaugh, T. L.; Garcia-Pintos, D.; Nørskov, J. K.; Bligaard, T.; Studt, F.; Campbell, C. T. A Benchmark Database for Adsorption Bond Energies to Transition Metal Surfaces and Comparison to Selected DFT Functionals. Surf. Sci. 2015, 640, 3644.

(14) Kepp, K. P. Benchmarking Density Functionals for Chemical Bonds of Gold. J. Phys. Chem. A 2017, 121 (9), 2022-2034.

(15) Jensen, K. P. Metal-Ligand Bonds of Second and Third Row d- Block Metals Characterized by Density Functional Theory. J. Phys. Chem. A 2009, 113, 1013310141.

(16) Kepp, K. P. Accuracy of Theoretical Catalysis from a Model of Iron-Catalyzed Ammonia Synthesis. Commun. Chem. 2018, 1 (1), 63.

(17) Jensen, K. P. Bioinorganic Chemistry Modeled with the TPSSh Density Functional. 
Inorg. Chem. 2008, 47, 10357-10365.

(18) Ahlrichs, R.; Bär, M.; Häser, M.; Horn, H.; Kölmel, C. Electronic Structure Calculations on Workstation Computers: The Program System Turbomole. Chem. Phys. Lett. 1989, 162 (3), 165-169.

(19) Turbomole 7.0. University of Karlsruhe and Forschungszentrum Karlsruhe GmbH 2012, p available from http://www.turbomole.com.

(20) Frisch, M. J.; Trucks, G. W.; Schlegel, H. B.; Scuseria, G. E.; Robb, M. A.; Cheeseman, J. R.; Scalmani, G.; Barone, V.; Petersson, G. A.; Nakatsuji, H.; et al. Gaussian 16, Revision A.03. 2016.

(21) Sierka, M.; Hogekamp, A.; Ahlrichs, R. Fast Evaluation of the Coulomb Potential for Electron Densities Using Multipole Accelerated Resolution of Identity Approximation. J. Chem. Phys. 2003, 118 (20), 9136-9148.

(22) Perdew, J. Density-Functional Approximation for the Correlation Energy of the Inhomogenous Electron Gas. Phys. Rev. B 1986, 33 (12), 8822-8824.

(23) Becke, A. D. Density-Functional Exchange-Energy Approximation with Correct Asymptotic-Behavior. Phys. Rev. A 1988, 38 (6), 3098-3100.

(24) Weigend, F.; Ahlrichs, R. Balanced Basis Sets of Split Valence, Triple Zeta Valence and Quadruple Zeta Valence Quality for H to Rn: Design and Assessment of Accuracy. Phys. Chem. Chem. Phys. 2005, 7 (18), 3297-3305.

(25) Jensen, K. P.; Roos, B. O.; Ryde, U. Performance of Density Functionals for First Row Transition Metal Systems. J. Chem. Phys. 2007, 126, 014103.

(26) Dunning, T. H. Gaussian Basis Sets for Use in Correlated Molecular Calculations. I. The 
Atoms Boron through Neon and Hydrogen. J. Chem. Phys. 1989, 90 (2), 1007-1023.

(27) Kramida, A.; Ralchenko, Y.; Reader, J.; NIST ASD Team (2018). NIST Atomic Spectra Database (ver. 5.5.6), [Online].

(28) Wolf, A.; Reiher, M.; Hess, B. A. The Generalized Douglas-Kroll Transformation. J. Chem. Phys. 2002, 117 (20), 9215-9226.

(29) Reiher, M. Douglas-Kroll-Hess Theory: A Relativistic Electrons-Only Theory for Chemistry. Theor. Chem. Acc. 2006, 116 (1-3), 241-252.

(30) Cheng, L.; Gauss, J.; Ruscic, B.; Armentrout, P. B.; Stanton, J. F. Bond Dissociation Energies for Diatomic Molecules Containing 3d Transition Metals: Benchmark ScalarRelativistic Coupled-Cluster Calculations for 20 Molecules. J. Chem. Theory Comput. 2017, 13 (3), 1044-1056.

(31) Grimme, S. Semiempirical Hybrid Density Functional with Perturbative Second-Order Correlation. J. Chem. Phys. 2006, 124 (3), 34108.

(32) Becke, A. D. A New Mixing of Hartree-Fock and Local Density-Functional Theories. J. Chem. Phys. 1993, 98 (2), 1372-1377.

(33) Yanai, T.; Tew, D. P.; Handy, N. C. A New Hybrid Exchange-correlation Functional Using the Coulomb-Attenuating Method (CAM-B3LYP). Chem. Phys. Lett. 2004, 393 (1), 51-57.

(34) Zhao, Y.; Truhlar, D. G. The M06 Suite of Density Functionals for Main Group Thermochemistry, Thermochemical Kinetics, Noncovalent Interactions, Excited States, and Transition Elements: Two New Functionals and Systematic Testing of Four M06Class Functionals and 12 Other Function. Theor. Chem. Acc. 2008, 120 (1-3), 215-241. 
(35) Perdew, J. P.; Burke, K.; Ernzerhof, M. Generalized Gradient Approximation Made Simple. Phys. Rev. Lett. 1996, 77 (18), 3865.

(36) Becke, A. D. Density-functional Thermochemistry. III. The Role of Exact Exchange. J. Chem. Phys. 1993, 98 (7), 5648-5652.

(37) Lee, C.; Yang, W.; Parr, R. G. Development of the Colle-Salvetti Correlation-Energy Formula into a Functional of the Electron Density. Phys. Rev. B 1988, 37 (2), 785-789.

(38) Stephens, P. J.; Devlin, F. J.; Chabalowski, C. F.; Frisch, M. J. Ab Initio Calculation of Vibrational Absorption and Circular Dichroism Spectra Using Density Functional Force Fields. J. Phys. Chem. 1994, 98 (45), 11623-11627.

(39) Hammer, B.; Hansen, L. B.; Nørskov, J. K. Improved Adsorption Energetics within Density-Functional Theory Using Revised Perdew-Burke-Ernzerhof Functionals. Phys. Rev. B 1999, 59 (11), 7413.

(40) Becke, A. D. Density-Functional Exchange-Energy Approximation with Correct Asymptotic Behavior. Phys. Rev. A 1988, 38 (6), 3098-3100.

(41) Slater, J. C. Quantum Theory of Molecular and Solids. Vol. 4: The Self-Consistent Field for Molecular and Solids; McGraw-Hill: New York, 1974.

(42) Vosko, S. H.; Wilk, L.; Nusair, M. Accurate Spin-Dependent Electron Liquid Correlation Energies for Local Spin Density Calculations: A Critical Analysis. Can. J. Phys. 1980, 58 (8), 1200-1211.

(43) Perdew, J. P. Density-Functional Approximation for the Correlation Energy of the Inhomogeneous Electron Gas. Phys. Rev. B 1986, 33 (12), 8822-8824.

(44) Adamo, C.; Barone, V. Toward Reliable Density Functional Methods without 
Adjustable Parameters: The PBE0 Model. J. Chem. Phys. 1999, 110 (13), 6158-6170.

(45) John Rumble. CRC Handbook of Chemistry and Physics, 98th Edition; CRC Handbook of Chemistry and Physics; CRC Press LLC, 2017.

(46) Grimme, S.; Antony, J.; Ehrlich, S.; Krieg, H. A Consistent and Accurate Ab Initio Parametrization of Density Functional Dispersion Correction (DFT-D) for the 94 Elements H-Pu. J. Chem. Phys. 2010, 132 (15), 154104.

(47) Wasserman, A.; Nafziger, J.; Jiang, K.; Kim, M.-C.; Sim, E.; Burke, K. The Importance of Being Inconsistent. Annu. Rev. Phys. Chem. 2017, 68 (1), 555-581.

(48) Lee, D.; Furche, F.; Burke, K. Accuracy of Electron Affinities of Atoms in Approximate Density Functional Theory. J. Phys. Chem. Lett. 2010, 1 (14), 2124-2129.

(49) Kepp, K. P. Energy vs. Density on Paths toward More Exact Density Functionals. Phys. Chem. Chem. Phys. 2018, 20 (11), 7538-7548.

(50) Sim, E.; Song, S.; Burke, K. Quantifying Density Errors in DFT. J. Phys. Chem. Lett. 2018, 9 (22), 6385-6392.

(51) Zhang, Y.; Yang, W. A Challenge for Density Functionals: Self-Interaction Error Increases for Systems with a Noninteger Number of Electrons. J. Chem. Phys. 1998, 109 (7), 2604-2608.

(52) Lundberg, M.; Siegbahn, P. E. M. Quantifying the Effects of the Self-Interaction Error in DFT: When Do the Delocalized States Appear? J. Chem. Phys. 2005, 122 (22), 224103.

(53) Ruzsinszky, A.; Perdew, J. P.; Csonka, G. I.; Vydrov, O. A.; Scuseria, G. E. Spurious Fractional Charge on Dissociated Atoms: Pervasive and Resilient Self-Interaction Error 
of Common Density Functionals. J. Chem. Phys. 2006, 125 (19), 194112.

(54) Cohen, A. J.; Mori-Sánchez, P.; Yang, W. Development of Exchange-Correlation Functionals with Minimal Many-Electron Self-Interaction Error. J. Chem. Phys. 2007, 126 (19), 191109.

(55) Xu, X.; Zhang, W.; Tang, M.; Truhlar, D. G. Do Practical Standard Coupled Cluster Calculations Agree Better than Kohn-Sham Calculations with Currently Available Functionals When Compared to the Best Available Experimental Data for Dissociation Energies of Bonds to 3d Transition Metals? J. Chem. Theory Comput. 2015, 11 (5), 2036-2052.

(56) Kepp, K. P. Trends in Strong Chemical Bonding in C2, CN, CN-, CO, N2, NO, NO+, and O2. J. Phys. Chem. A 2017, 121 (47), 9092-9098.

(57) Kepp, K. P. Comment on "Density Functional Theory Is Straying from the Path toward the Exact Functional.” Science 2017, 356 (6337), 496-497.

(58) Medvedev, M. G.; Bushmarinov, I. S.; Sun, J.; Perdew, J. P.; Lyssenko, K. A. Density Functional Theory Is Straying from the Path toward the Exact Functional. Science 2017, 355 (6320), 49-52.

(59) Kim, M.-C.; Sim, E.; Burke, K. Understanding and Reducing Errors in Density Functional Calculations. Phys. Rev. Lett. 2013, 111 (7), 73003.

(60) Perdew, J. P. Jacob’s Ladder of Density Functional Approximations for the ExchangeCorrelation Energy. AIP Conf. Proc. 2001, 577 (1), 1-20.

(61) Jensen, K.; Ryde, U. Theoretical Prediction of the Co-C Bond Strength in Cobalamins. J. Phys. Chem. A 2003, 155, 7539-7545. 
(62) Neese, F. A Critical Evaluation of DFT, Including Time-Dependent DFT, Applied to Bioinorganic Chemistry. JBIC J. Biol. Inorg. Chem. 2006, 11 (6), 702-711.

(63) Mori-Sánchez, P.; Cohen, A. J.; Yang, W. Many-Electron Self-Interaction Error in Approximate Density Functionals. J. Chem. Phys. 2006, 125 (20), 201102.

(64) Bao, J. L.; Gagliardi, L.; Truhlar, D. G. Self-Interaction Error in Density Functional Theory: An Appraisal. J. Phys. Chem. Lett. 2018, 9 (9), 2353-2358.

(65) Cohen, A. J. Insights into Current Limitations of Density Functional Theory. Science 2011, 792 (2008), 792-794.

(66) Cohen, A. J.; Mori-Sánchez, P.; Yang, W. Challenges for Density Functional Theory. Chem. Rev. 2011, 112 (1), 289-320.

(67) Jiang, W.; DeYonker, N. J.; Wilson, A. K. Multireference Character for 3d TransitionMetal-Containing Molecules. J. Chem. Theory Comput. 2012, 8 (2), 460-468. 
TOC graphic

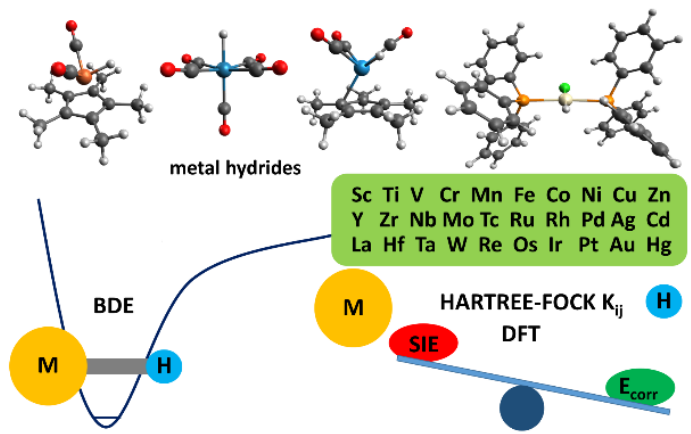

